\title{
Contrast-Enhanced Ultrasound Evaluation of Mifepristone for Treatment of Low-Risk Cesarean Scar Pregnancy
}

\author{
Xi Xiong $\mathbb{D}^{1},{ }^{1}$ Chun-yan Gao, ${ }^{1}$ De-mei Ying, ${ }^{1}$ Ping Yan, ${ }^{1}$ Zhi-jia Zhang, ${ }^{2}$ Na Kuang, \\ Hong-ju Tian, ${ }^{1}$ Li Luo, ${ }^{1}$ Shu-yu Long, ${ }^{1}$ and Zheng-qiong Chen ${ }^{1}{ }^{1}$ \\ ${ }^{1}$ Department of Obstetrics and Gynecology, Second Clinical Medical College of Army Medical University, \\ Chongqing 400037, China \\ ${ }^{2}$ Department of Clinical Laboratory, Second Clinical Medical College of Army Medical University, \\ Chongqing 400037, China
}

Correspondence should be addressed to Zheng-qiong Chen; chenzhengqiong75@163.com

Received 22 June 2020; Revised 9 October 2020; Accepted 18 October 2020; Published 31 October 2020

Academic Editor: Wei Li

Copyright (c) $2020 \mathrm{Xi}$ Xiong et al. This is an open access article distributed under the Creative Commons Attribution License, which permits unrestricted use, distribution, and reproduction in any medium, provided the original work is properly cited.

Purpose. The effect of mifepristone for treatment of low-risk cesarean scar pregnancy (CSP) was monitored by contrast-enhanced ultrasound (CEUS). Methods. Data were collected from 23 CSP patients with a 10-point risk score $<5$ (low-risk CSP) and from 23 intrauterine pregnancy (IUP) patients with a scar from a previous cesarean delivery. All patients were prescribed $75 \mathrm{mg}$ mifepristone daily for 2 days and underwent transvaginal CEUS before and after administration of mifepristone. On the third day, uterine curettage was performed after transvaginal CEUS. Arrival time (AT), peak intensity (PI), and area under the curve (AUC) around the gestational sac were monitored by CEUS before and after application of mifepristone, and the rate of effective treatment was compared between the two patient groups. Results. No patients experienced side effects from either the CEUS procedure or the mifepristone treatment. Changes in AT, PI, and AUC index from before vs. after mifepristone treatment did not differ significantly between the two groups (all $p$ values $>0.05$ ). There was also no significant difference in the rate of effective treatment between the two groups (95.65\% in the CSP group vs. $100 \%$ in the IUP group; $p>0.05)$. Conclusions. Based on monitoring by CEUS, the effect of mifepristone in low-risk CSP was comparable to that in IUP.

\section{Introduction}

Cesarean scar pregnancy (CSP), or implantation of the gestational sac in a hysterotomy scar, is a rare but serious complication that can occur in a subsequent pregnancy after cesarean delivery [1]; it is especially concerning in China [2]. Prenatal diagnosis of CSP is based on the presence of a gestational sac at the site of the previous uterine incision and the presence of an empty uterine cavity and cervix and thin myometrium adjacent to the bladder [3]. The severity of CSP has been found to correlate with clinical and sonographic characteristics including the implantation site, blood flow around the gestational sac, timing within gestation, and number of previous cesarean deliveries $[4,5]$. Numerous management options for CSP have been evaluated based on case series, including laparoscopy, uterine artery embolization (UAE), and high-intensity focused ultrasound (HIFU) [6-8]. However, no standardized diagnostic or management guidelines have been published [9].

Our group has developed and validated a scoring system to rate the severity of CSP on a 10-point scale based on clinical indicators including thickness of the myometrium at uterine incision, grading of blood flow, fetal heartbeat, location of the gestational sac, maximal diameter of the gestational sac, and number of previous cesarean sections [10]. To validate this scoring system, patients were assigned a risk score based on these indicators, and treatment modalities employed were then assessed in relation to risk scores. Results showed that patients with CSP risk scores lower than 5 were significantly less likely to need invasive salvage treatments compared to higher-risk patients [10]. 
Mifepristone is the most commonly used progesterone antagonist [11]. In addition to producing prostaglandins to accelerate the degeneration and necrosis of villi, mifepristone can also reduce the vascular endothelial growth factor in decidual tissue, thereby reducing blood supply to the embryo and bringing about termination of the pregnancy [12]. A case report showed that the approach with mifepristone for treatment of CSP may be a safer and less invasive method [13]. By contrast, another study suggested that mifepristone is not very effective in the treatment of CSP [14]. Therefore, there are no commonly accepted clinical management guidelines on the use of mifepristone for CSP.

Contrast-enhanced ultrasound (CEUS) is a safe, widely available, and relatively inexpensive imaging technique that uses dedicated imaging ultrasound sequences and FDAapproved contrast microbubbles, permitting high diagnostic accuracy [15]. CEUS is a convenient method for diagnosis of CSP, has excellent spatial and temporal resolution, and can be used for quantitative assessment of microcirculation perfusion of the gestational sac [16]. Building on findings from our previous work, we sought to quantitatively analyze changes in microcirculation around the gestational sac using CEUS in order to evaluate the efficacy of mifepristone in the treatment of low-risk CSP.

\section{Materials and Methods}

Data were prospectively collected between July 2018 and March 2019 from patients seen in the department of obstetrics and gynecology of the Second Clinical Medical College of Army Medical University of China. Participants included a group of patients with low-risk CSP (10-point risk score $<5, n=23$ ) and an equal-sized control group of patients having intrauterine pregnancy (IUP) with scar and electing to terminate pregnancy. Patients were excluded from the study if they had serious diseases of vital organs such as the heart, kidney, and lungs. Diagnosis of CSP was confirmed by review of sonographic images. In accordance with the " 2013 revision of the Declaration of Helsinki," all study participants gave written informed consent regarding study procedures and treatment modalities after the procedures had been fully explained to them.

We assessed relevant demographic and clinical characteristics including age, parity, gestational age, BMI, and remnant myometrial thickness. All patients were prescribed $75 \mathrm{mg}$ mifepristone daily for 2 days and underwent transvaginal CEUS before and after administration of mifepristone (Figures 1 and 2). On the third day, uterine curettage was performed after transvaginal CEUS.

2.1. CEUS Examination. All patients were examined by two obstetric ultrasound technicians with at least 5 years of experience. All ultrasound examinations were conducted using a Philips IU-22 system (Philips Electronics N.V., Amsterdam, Netherlands) with a $5-9 \mathrm{MHz}$ transvaginal transducer. A $21 \mathrm{G}$ trocar was used to puncture the cubital vein and establish a venous channel. Next, $2.5 \mathrm{~mL}$ of the contrast agent was injected, and $5 \mathrm{~mL}$ of $0.9 \%$ normal saline was used for tube washing. When the contrast agent was injected, the patient began holding their breath and took a shallow breath when required or alternatively continued with slow shallow breathing. All patients were trained in the required breathing regime before the contrast process. Two minutes of ultrasound data were recorded and saved for analysis. The lesion area with the most evident enhancement was identified as the region of interest (ROI), with ROIs set as $5 \mathrm{~mm}$ diameter circles and remaining unchanged. The ROIs were located at the embryo decidua basalis. Related parameters obtained through the time intensity curve (TIC) included arrival time (AT, the time from injection of the agent to the point when the first contrast bubbles appeared in the gestational sac), peak intensity (PI, the maximal intensity of the TIC), and area under the curve around the gestational sac (AUC, the area under the TIC) [17].

Operations were ceased if vaginal bleeding exceeded $300 \mathrm{~mL}$, in which case uterine balloons were employed for temporary hemostasis. It is recommended that UAE be performed if bleeding exceeds $500 \mathrm{~mL}$. All study patients were followed up for at least one month following the study procedure; assessments included the serum $\beta$-hCG level and presence of abdominal pain, vaginal bleeding, and fever every month.

2.2. Evaluation of Curative Effects. Curative effects were assessed at three months following treatment and were rated as follows:

2.2.1. Excellent Curative Effect. Ultrasound showed no residual gestational tissue; the patient had no abdominal pain, vaginal bleeding, or fever, and the serum $\beta$-hCG level decreased and returned to normal in three months.

2.2.2. Moderate Curative Effect. Ultrasound results showed residual gestational tissue; the patient had abdominal pain, vaginal bleeding, or fever, and the serum $\beta$-hCG level was decreased. After recurettage or pharmaceutical treatment, ultrasound showed no residual gestational tissue, there were no symptoms such as abdominal pain, vaginal bleeding, or fever, and the serum $\beta$-hCG level returned to normal within three months.

2.2.3. Poor Curative Effect. Ultrasound showed residual gestational tissue; the patient had abdominal pain, vaginal bleeding, or other symptoms, and the serum $\beta$-hCG level may have increased or decreased but did not decrease to normal levels. After recurettage or pharmaceutical treatment, ultrasound showed that the residual gestational tissue had persisted or grown. Patients had persistent vaginal bleeding or abdominal pain and needed further treatment such as laparoscopic surgery or UAE.

The total effective treatment rate was defined as the number of patients for whom treatment was rated as excellent or moderate divided by the total number of patients in each study group and expressed as a percentage. 


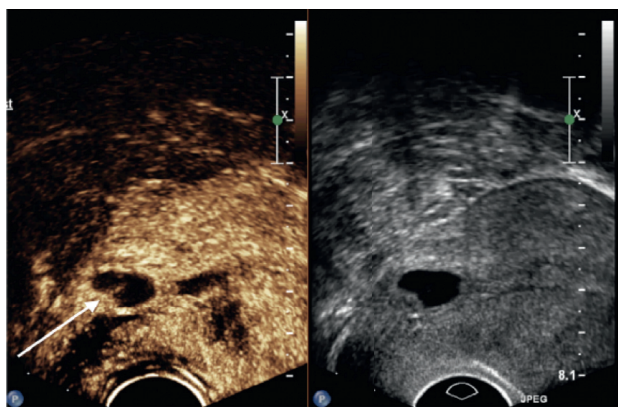

(a)

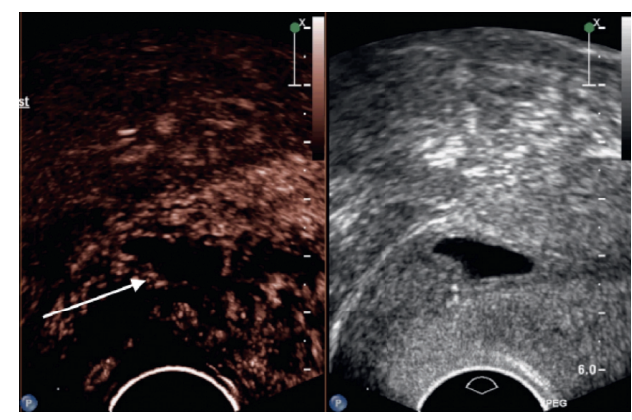

(b)

FIGURE 1: Findings from transvaginal contrast-enhanced ultrasonography before and after mifepristone administration in patients with lowrisk cesarean scar pregnancy. (a) Before mifepristone treatment. (b) After mifepristone treatment.

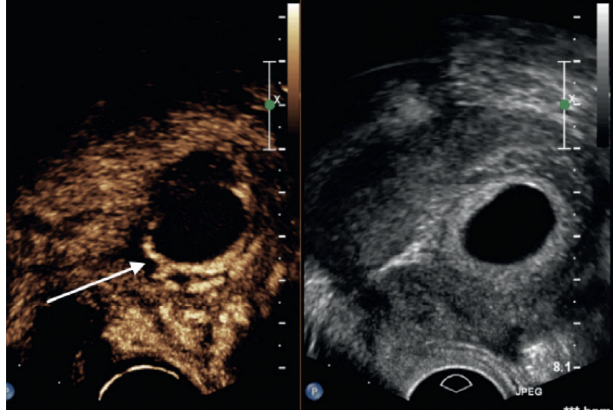

(a)

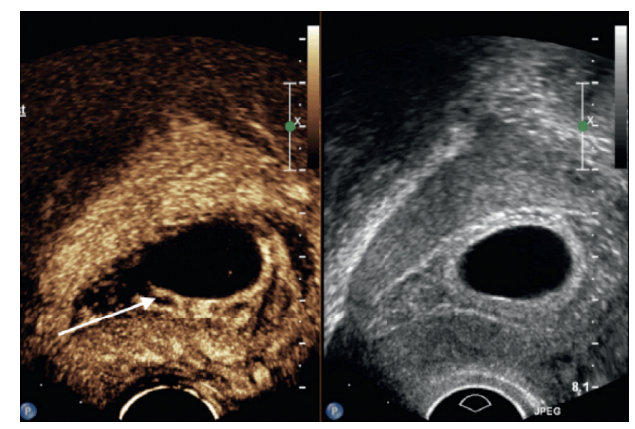

(b)

Figure 2: Findings from transvaginal contrast-enhanced ultrasonography before and after mifepristone administration in patients with intrauterine pregnancy. (a) Before mifepristone treatment. (b) After mifepristone treatment.

2.3. Statistical Analysis. Analyses were performed using SPSS software version 16.0 (IBM, Armonk, NY, USA). All variables are presented as mean \pm standard deviation. The paired $t$-test was used to compare patient characteristics between the CSP and IUP groups, to compare perfusion parameters as assessed by CEUS before vs. after mifepristone treatment, and to compare changes in perfusion parameters and curative effects between the two study groups. The chisquared test was used to verify the efficacy of mifepristone. A $p$ value of $<0.05$ was considered statistically significant.

\section{Results}

3.1. Patient Demographics and Clinical Characteristics. Patient characteristics for the CSP and IUP groups are shown in Table 1. Mean thickness of the lower uterine segment was significantly higher among women with IUP $(5.72 \pm 1.65 \mathrm{~mm}$ vs. $2.60 \pm 1.20, p \leq 0.001)$. There were no other significant differences between the two patient groups.

There were 16 patients with fetal heart activity in the CSP group and 15 patients in the IUP group. Two days after misoprostol administration, there were 9 patients without fetal heart activity detected in the CSP group and 7 patients without fetal heart activity in the IUP group (difference between the two groups not statistically significant, $\left.\chi^{2}=2.000 ; p=0.368\right)$.
Three patients in the CSP group reported pain without bleeding, 7 patients presented with bleeding but did not report pain, and 7 patients presented with both pain and bleeding. In the IUP group, 4 patients reported pain with no bleeding, 6 patients presented with bleeding but did not report pain, and 6 patients presented with both pain and bleeding.

3.2. CEUS Findings. CEUS perfusion quantification values for the two study groups before and after mifepristone treatment are shown in Table 2. In the CSP group, before mifepristone treatment, AT is $18.42 \pm 3.38$ (s), PI is $17.68 \pm 2.84(\mathrm{~dB})$, and AUC is $1011.03 \pm 194.53$, and after mifepristone treatment, AT is $13.39 \pm 1.98$ (s), PI is $14.48 \pm 2.81(\mathrm{~dB})$, and AUC is $800.33 \pm 109.41$. In IUP group, before mifepristone treatment, AT is $18.71 \pm 2.01(\mathrm{~s}), \mathrm{PI}$ is $17.85 \pm 2.61(\mathrm{~dB})$, and AUC is $1041.76 \pm 168.14$, and after mifepristone treatment, AT is $14.06 \pm 2.85$ (s), PI is $15.47 \pm 2.44(\mathrm{~dB})$, and AUC is $878.49 \pm 162.23$. Based on TIC analysis, AT, PI, and AUC around the gestational sac were significantly lower in both study groups after mifepristone treatment than before $(p<0.05$; Table 2$)$.

Forty-eight hours following administration of mifepristone, in the CSP group, AT, PI, and AUC changes in blood flow around the gestational sac are $5.03 \pm 2.97$ (s), 
TABle 1: Patient characteristics.

\begin{tabular}{|c|c|c|c|c|}
\hline Characteristic & $\operatorname{CSP}(n=23)$ & IUP $(n=23)$ & $t$ & $p$ \\
\hline Maternal age (years) & $32.26 \pm 3.99$ & $32.43 \pm 3.78$ & 0.152 & 0.880 \\
\hline BMI & $23.12 \pm 2.92$ & $22.37 \pm 3.96$ & -0.726 & 0.471 \\
\hline Gravidity & $4.29 \pm 1.43$ & $4.61 \pm 1.47$ & 0.507 & 0.615 \\
\hline Parity & $1.47 \pm 0.51$ & $1.52 \pm 0.51$ & 0.289 & 0.774 \\
\hline Diameter of gestational sac (mm) & $20.40 \pm 7.90$ & $21.37 \pm 11.64$ & 0.331 & 0.742 \\
\hline Previous cesarean deliveries (times) & $1.47 \pm 0.51$ & $1.52 \pm 0.51$ & 0.289 & 0.774 \\
\hline Thickness of the lower uterine segment (mm) & $2.60 \pm 1.20$ & $5.72 \pm 1.65$ & 7.341 & $\leq 0.001$ \\
\hline
\end{tabular}

CSP, cesarean scar pregnancy; IUP, intrauterine pregnancy.

TABLE 2: CEUS perfusion quantification before and after mifepristone treatment in CSP and IUP patients.

\begin{tabular}{|c|c|c|c|c|c|}
\hline Group & Parameter & Before mifepristone treatment & After mifepristone treatment & $t$ & $p$ \\
\hline \multirow{3}{*}{$\operatorname{CSP}(n=23)$} & $\mathrm{AT}(\mathrm{s})$ & $18.42 \pm 3.38$ & $13.39 \pm 1.98$ & 8.117 & $\leq 0.001$ \\
\hline & PI $(\mathrm{dB})$ & $17.68 \pm 2.84$ & $14.48 \pm 2.81$ & 6.446 & $\leq 0.001$ \\
\hline & AUC & $1011.03 \pm 194.53$ & $800.33 \pm 109.41$ & 8.341 & $\leq 0.001$ \\
\hline \multirow{3}{*}{ IUP $(n=23)$} & $\mathrm{AT}(\mathrm{s})$ & $18.71 \pm 2.01$ & $14.06 \pm 2.85$ & 7.208 & $\leq 0.001$ \\
\hline & PI (dB) & $17.85 \pm 2.61$ & $15.47 \pm 2.44$ & 4.411 & $\leq 0.001$ \\
\hline & AUC & $1041.76 \pm 168.14$ & $878.49 \pm 162.23$ & 10.961 & $\leq 0.001$ \\
\hline
\end{tabular}

AT, arrival time; AUC, the area under the time intensity curve; CEUS, contrast-enhanced ultrasonography; CSP, cesarean scar pregnancy; IUP, intrauterine pregnancy; PI, peak intensity.

$0.01 \pm 1.78(\mathrm{~dB})$, and $210.69 \pm 121.14$; in the IUP group, AT, $\mathrm{PI}$, and AUC changes in blood flow around the gestational sac are $4.65 \pm 3.09(\mathrm{~s}), 0.15 \pm 1.53(\mathrm{~dB})$, and 169.26 \pm 74.06 . Based on TIC analysis, changes in blood flow around the gestational sac, including AT, PI, and AUC, did not differ significantly between the two groups (Table 3 ).

3.3. Evaluation of Curative Effects. In the IUP group, no patients had vaginal bleeding $>500 \mathrm{~mL}$ during uterine $\mathrm{cu}$ rettage. One week after uterine curettage, ultrasound showed no residual gestational tissue and no abdominal pain, vaginal bleeding, or fever, and serum $\beta$-hCG levels returned to normal in three months in all patients. Thus, the total effective treatment rate was $100 \%$ in the IUP group.

In the CSP group, two patients had vaginal bleeding $>500 \mathrm{~mL}$ during uterine curettage; bleeding was significantly reduced following administration of $1 \mathrm{~mL}$ oxytocin during the operation, and no further treatment was needed. One week following the operation, 6 patients had residual gestational tissue, minimal vaginal bleeding, and no abdominal pain. These patients were instructed to take mifepristone orally. Among them, 2 patients underwent recurettage because there had been no evident reduction of gestational tissue. After recurettage, ultrasound showed no residual gestational tissue, there was no vaginal bleeding, and serum $\beta$-hCG levels returned to normal. One patient underwent laparoscopic resection after conservative treatment for 1 month because of persistent vaginal bleeding, continuous enlargement of residual gestational tissue, and disappearance of local muscular layer (Figure 2); there was no significant decrease in the serum $\beta$-hCG level in this patient. In the remaining 3 CSP patients with no abdominal pain, residual gestational tissue gradually shrank and returned to normal within one month, and serum $\beta$-hCG levels returned
TABLE 3: Changes in blood flow around the gestational sac before vs. after mifepristone treatment in CSP and IUP patients.

\begin{tabular}{lcccc}
\hline Parameter & CSP $(n=23)$ & IUP $(n=23)$ & $t$ & $p$ \\
\hline AT (s) & $5.03 \pm 2.97$ & $4.65 \pm 3.09$ & -0.424 & 0.674 \\
PI (dB) & $0.01 \pm 1.78$ & $0.15 \pm 1.53$ & -1.115 & 0.271 \\
AUC & $210.69 \pm 121.14$ & $169.26 \pm 74.06$ & -1.399 & 0.169 \\
\hline
\end{tabular}

AT, arrival time; AUC, the area under the time intensity curve; CSP, cesarean scar pregnancy; IUP, intrauterine pregnancy; PI, peak intensity.

to normal within three months. The total effective treatment rate among CSP patients was thus 95.65\% (Table 4). The effective treatment rate did not differ significantly between the two study groups $\left(\chi^{2}=4.000 ; p=0.261\right)$.

\section{Discussion}

With continuing advances in research on CSP, it is now understood that the risk posed by CSP is affected by many factors, including the number of previous cesarean deliveries, the position of implantation of the gestational sac, and the timing within gestation $[4,5]$. While numerous management options for CSP have been identified and evaluated, no standardized diagnostic or management guidelines have been developed $[8,9,18]$. Therefore, it is crucial to make an accurate diagnosis and to provide prompt therapy to avoid potentially catastrophic complications.

In our previous study evaluating the utility of a CSP risk scoring system to predict appropriate treatment, we found mifepristone combined with uterine curettage to be the optimal treatment for low-risk patients (those with a risk score $<5)$ [10]. In the present study, we found no significant differences in maternal age, BMI, gravidity, parity, or gestational days between CSP and IUP patients, but average muscle layer thickness in the CSP group was less than half of that of the normal pregnancy group. Our finding of low 
TABLE 4: Comparison of curative effects between CSP and IUP patients.

\begin{tabular}{lccccc}
\hline Group & $n$ & Excellent curative effect & Moderate curative effect & Poor curative effect & Effective treatment rate (\%) \\
\hline CSP & 23 & 16 & 6 & 1 & 95.65 \\
IUP & 23 & 23 & 0 & 0 & 100 \\
\hline
\end{tabular}

CSP, cesarean scar pregnancy; IUP, intrauterine pregnancy.

remnant myometrial thickness in CSP is consistent with results from previous studies [9] and may stem from erosion of the muscular layer of the gestational sac when it is implanted in the scar, resulting in thinning of the gestational sac.

The effectiveness of high-dose mifepristone for abortion has been well-established [19-21]. Mifepristone influences the human endometrium during the luteal phase by reducing stromal edema, increasing venular diameter, and causing erythrocyte and leukocyte diapedesis and focal hemorrhage and degeneration of the stromal extracellular matrix. Through these mechanisms, eventual degradation of the endometrium is initiated, leading to termination of pregnancy.

CEUS has become a widely available and well-accepted imaging modality in recent years. By overcoming some of the limitations of conventional ultrasonography, CEUS creates a significant opportunity for visualization of the microcirculation [22]. Findings from the present study show that perfusion parameters around the gestational sac were significantly reduced following mifepristone treatment in both low-risk CSP patients and women with IUP. Accordingly, it appears that mifepristone brings about medical abortion in part through reducing microcirculation of the gestational sac by acting on endometrial vessels. In addition, changes in microcirculation of the gestational sac did not differ significantly between the two study groups, suggesting that mifepristone has the same effect on pregnancy termination in low-risk CSP as in normal pregnancy. Furthermore, both groups achieved similar curative effects through mifepristone combined with curettage. Accordingly, more aggressive treatments such as laparoscopy, hysteroscopy, and UAE can be avoided through the use of mifepristone combined with curettage in low-risk CSP patients. This conclusion is consistent with findings from Fu et al. [23] and suggests that personalizing treatment options based on the patient's condition can reduce the physical and mental impact of treatment on patients while also reducing the cost of their care.

Several limitations to our study should be acknowledged. First, this study was conducted at a single center, and our results should be confirmed in larger multicenter studies before being applied more widely in clinical practice. Second, we did not look at long-term outcomes such as recurrent ectopic pregnancy or subsequent fertility. We recommend that future studies examine these outcomes in order to develop a richer understanding of the long-term safety and risks of mifepristone for treatment of low-risk CSP.

In conclusion, based on monitoring by CEUS, the effect of mifepristone in low-risk CSP was comparable to that in IUP, and combined with uterine curettage, we found this treatment was safe and effective in patients with low-risk CSP. In this patient population, such a treatment course can be used to avoid more aggressive treatments such as laparoscopy, hysteroscopy, and UAE.

\section{Data Availability}

The data used to support the findings of this study are available from the corresponding author upon request.

\section{Conflicts of Interest}

The authors declare that they have no conflicts of interest.

\section{Authors' Contributions}

Xi Xiong, Chun-yan Gao, and De-mei Ying have contributed equally to this paper.

\section{Acknowledgments}

The authors thank LetPub (http://www.letpub.com) for its linguistic assistance and scientific consultation during the preparation of this manuscript. Funding for this research was provided by the Clinical Research Foundation of the Second Clinical Medical College of Army Medical University (2016YLC23), and the Technological Innovation and Application Development Project of the Chongqing Science and Technology Bureau (stc2019jscx-msxmX0269), with key project support from the Second Affiliated Hospital of Army Medical University (2019JSLC011).

\section{References}

[1] I. E. Timor-Tritsch, A. Monteagudo, R. Santos, T. Tsymbal, G. Pineda, and A. A. Arslan, "The diagnosis, treatment, and follow-up of cesarean scar pregnancy," American Journal of Obstetrics and Gynecology, vol. 207, p. 44, 2012.

[2] L. Luo, X. Ruan, C. Li, S. Chen, Q. Hu, and A. O. Mueck, "Early clinical features and risk factors for cesarean scar pregnancy: a retrospective case-control study," Gynecological Endocrinology, vol. 35, no. 4, pp. 337-341, 2019.

[3] F. D'Antonio, J. Palacios-Jaraquemada, P. S. Lim et al., "Counseling in fetal medicine: evidence-based answers to clinical questions on morbidly adherent placenta," Ultrasound in Obstetrics \& Gynecology, vol. 47, no. 3, pp. 290-301, 2016.

[4] X. Xiong, X. M. Zhang, P. Yan, C. Y. Gao, and Q. L. Sun, "The value of ultrasound for early classification in cesarean scar pregnancy," International Journal of Clinical and Experimental Medicine, pp. 17267-17273, 2016.

[5] O. Grechukhina, U. Deshmukh, L. Fan et al., "Cesarean scar pregnancy, incidence, and recurrence," Obstetrics \& Gynecology, vol. 132, no. 5, pp. 1285-1295, 2018.

[6] S. Tanigaki, C. Nagata, K. Ueno et al., "Successful treatment of caesarean scar pregnancies by local treatment only," Obstetrics 
and Gynecology International, vol. 2017, Article ID 9543570, 2017.

[7] I. E. Timor-Tritsch, N. Khatib, A. Monteagudo, J. Ramos, R. Berg, and S. Kovács, "Cesarean scar pregnancies," Journal of Ultrasound in Medicine, vol. 34, no. 4, pp. 601-610, 2015.

[8] J. Xiao, Z. Shi, J. Zhou et al., "Cesarean scar pregnancy: comparing the efficacy and tolerability of treatment with highintensity focused ultrasound and uterine artery embolization," Ultrasound in Medicine \& Biology, vol. 43, no. 3, pp. 640-647, 2017.

[9] P. K. Birch, E. Hoffmann, R. C. Larsen, and H. S. Nielsen, "Cesarean scar pregnancy: a systematic review of treatment studies," Fertility and Sterility, vol. 105, no. 4, pp. 958-967, 2016.

[10] Q.-L. Sun, X.-H. Wu, L. Luo, D.-M. Ying, Y. Yang, and Z.-Q. Chen, "Characteristics of women with mixed mass formation after evacuation following uterine artery chemoembolization for cesarean scar pregnancy," Archives of Gynecology and Obstetrics, vol. 297, no. 4, pp. 1059-1066, 2018.

[11] C. Devane, R. M. Renner, S. Munro et al., "Implementation of mifepristone medical abortion in Canada: pilot and feasibility testing of a survey to assess facilitators and barriers," Pilot and Feasibility Studies, vol. 8, no. 5, p. 126, 2019.

[12] D. Ghosh, P. Kumar, and J. Sengupta, "Effect of early luteal phase administration of mifepristone (RU486) on leukaemia inhibitory factor, transforming growth factor beta and vascular endothelial growth factor in the implantation stage endometrium of the rhesus monkey," Journal of Endocrinology, vol. 157, no. 1, pp. 115-125, 1998.

[13] E. Kalampokas, I. Boutas, K. Panoulis, and T. Kalampokas, "Novel medical therapy of cesarean scar pregnancy with a viable embryo combining multidose methotrexate and mifepristone: a case report," Medicine, vol. 94, no. 41, Article ID e1697, 2015.

[14] Y. Y. Li, Z. Y. Yin, S. Li et al., "Comparison of transvaginal surgery and methotrexate/mifepristone-combined transcervical resection in the treatment of cesarean scar pregnancy," European Review for Medical and Pharmacological Sciences, vol. 21, no. 12, pp. 2957-2963, 2017.

[15] I. Durot, S. R. Wilson, and J. K. Willmann, "Contrast-enhanced ultrasound of malignant liver lesions," Abdominal Radiology, vol. 43, no. 4, pp. 819-847, 2018.

[16] X. Xiong, P. Yan, C. Gao, Q. Sun, and F. Xu, "The value of contrast-enhanced ultrasound in the diagnosis of cesarean scar pregnancy," BioMed Research International, vol. 2016, Article ID 4762785, 2016.

[17] S. J. Zhang, J. Du, R. H. Tian, S. W. Xie, F. H. Li, and Z. Li, "Assessment of the use of contrast enhanced ultrasound in guiding microdissection testicular sperm extraction in nonobstructive azoospermia," BMC Urology, vol. 18, no. 1, p. 48, 2018.

[18] S. Y. Kim, S. R. Yoon, M. J. Kim, J. H. Chung, M. Y. Kim, and S. W. Lee, "Cesarean scar pregnancy; Diagnosis and management between 2003 and 2015 in a single center," Taiwanese Journal of Obstetrics and Gynecology, vol. 57, no. 5, pp. 688-691, 2018.

[19] World Health Organization Task Force on Post-ovulatory Methods for Fertility Regulation, "Lowering the doses of mifepristone and gameprost for early abortion: a randomised controlled trial," BJOG, vol. 108, pp. 738-742, 2001.

[20] C. Papp, F. Schatz, G. Krikun, V. Hausknecht, and C. J. Lockwood, "Biological mechanisms underlying the clinical effects of mifepristone (RU 486) on the endometrium," Early Pregnancy, vol. 4, no. 4, pp. 230-239, 2000.
[21] L.-P. Song, S.-Y. Tang, C.-L. Li, L.-J.-G.-Y.-K. Zhou, and X.-T. Mo, "Early medical abortion with self-administered low-dose mifepristone in combination with misoprostol," Journal of Obstetrics and Gynaecology Research, vol. 44, no. 9, pp. 1705-1711, 2018.

[22] M. Sperandeo, G. Rea, M. A. Grimaldi, F. Trovato, L. M. C. Dimitri, and V. Carnevale, "Contrast-enhanced ultrasound does not discriminate between community acquired pneumonia and lung cancer," Thorax, vol. 72, no. 2, pp. 178-180, 2017.

[23] L. P. Fu, "Therapeutic approach for the cesarean scar pregnancy," Medicine, vol. 97, no. 18, Article ID e0476, 2018. 